\title{
Static model for a 3-DOF underactuated finger
}

\section{F. Guay and C. Gosselin}

Laboratoire de robotique, Département de génie mécanique, Université Laval, Québec, Canada

Received: 27 February 2010 - Revised: 30 June 2010 - Accepted: 19 August 2010 - Published: 8 February 2011

\begin{abstract}
This paper introduces a static model of a three-degree-of-freedom underactuated finger. The model includes all static forces, namely actuation forces, return forces and gravity. All geometric and static parameters can be freely changed (pulley radius, member's mass, etc.). Hence, the model allows complete static simulations to be performed and it can also be used for numerical optimization.
\end{abstract}

This paper was presented at the IFToMM/ASME International Workshop on Underactuated Grasping (UG2010), 19 August 2010, Montréal, Canada.

\section{Introduction}

Governmental investment in prosthetic research has increased significantly over the last decade, the most notable initiative being DARPA's Revolutionizing Prosthetics program in the US (Belfiore, 2009).

One of the most sought and important criterion is to develop a hand that looks and feels natural (Belfiore, 2009). That is the person who wears and uses the prosthesis doesn't feel out of place in public. This criterion is extremely important for that the person who is to wear the prosthesis will not prematurely discard it to never wear it again. Therefore, a prosthetic hand should have 5 fingers each including 3 phalanges.

A possible approach is to develop a hand in which each finger joint is independently actuated. Even with simplifications such as modelling the finger's joints as revolute joints, this leads to a total of 15 actuators which have to be powered and controlled. The problem encountered in such an approach is the integration of such a large number of parts, the amount of time and effort for someone who is not likely technically inclined to master and use this complex assembly, the reliability and ruggedness of such an assembly and, often the problem that will be determinant, the combined weight and wearability of such a system.

Therefore, underactuation is a very attractive alternative. Underactuation is the property of a mechanism that has fewer

Correspondence to: F. Guay

(francois.guay.2@ulaval.ca) degrees of actuation than degrees of freedom. The control of the degrees of freedom is achieved by mechanical intelligence.

Several underactuated fingers have been proposed in the literature (see for instance Birglen et al., 2008) for a literature survey). Many of the proposed fingers use tendon-based transmissions and a single degree of actuation. This principle is studied here and a finger model is proposed.

\section{The finger model}

The model proposed in this article considers the following torque vectors: $\tau_{a}$, the actuation which makes the finger curl, $\tau_{r}$, the spring action which makes the finger flex (open), $\tau_{f}$, the friction forces and, an action never considered before, $\tau_{g}$, the gravitational forces. $\tau_{a}$ and $\tau_{r}$ cannot be completely specified since we will impose a torque at only one joint of the finger. On the other hand, $\tau_{f}$ and $\tau_{g}$ can be fully specified for a given configuration of the finger. In order to model the behaviour of the finger, we will combine all four torque vectors. Figure 1 shows the components of these vectors and their position on the finger.

\section{Underactuation}

We begin by using, as a basis, the underactuation model proposed in Birglen et al. (2008); Allen-Demers et al. (2009) and adapt it to a typical finger used in a prosthesis. Here the only adaptation is to realise that our finger has 3 phalanges which are underactuated. 


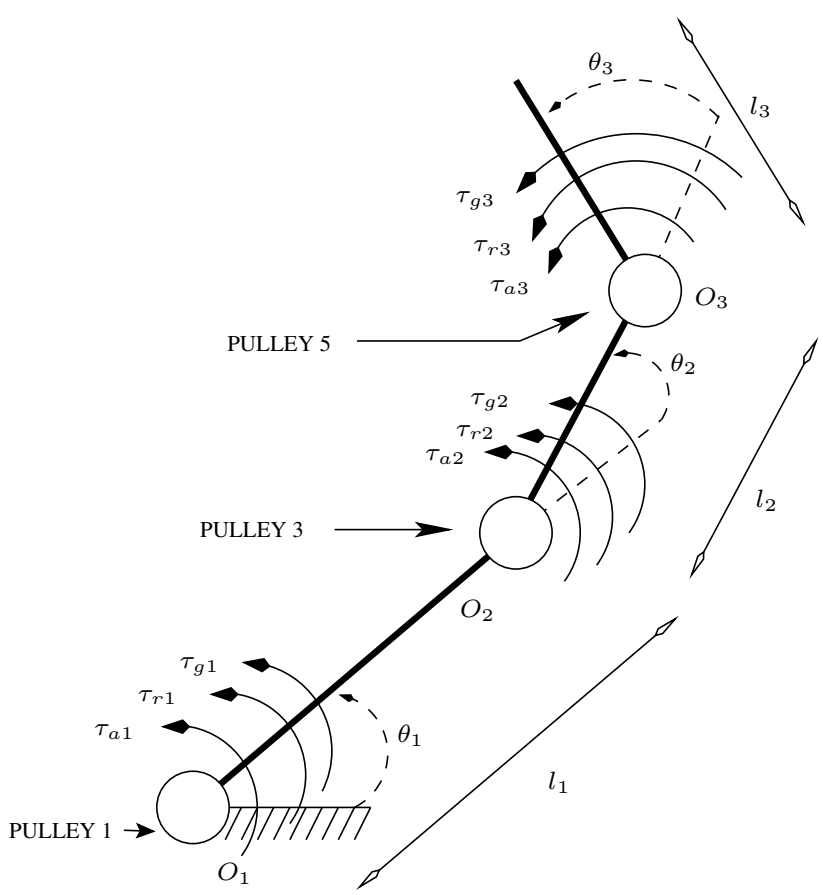

Figure 1. Finger with corresponding variables.

We equate the input and output virtual powers acting on the finger and obtain (Birglen et al., 2008):

$t^{T} \omega_{a}=\sum_{i=1}^{3} \xi_{i} \circ \zeta_{i}$

where

$\boldsymbol{t}=\left[\begin{array}{c}T_{a} \\ 0 \\ 0\end{array}\right], \quad \boldsymbol{\omega}_{a}=\left[\begin{array}{c}\dot{\theta}_{1} \\ \dot{\theta}_{2} \\ \dot{\theta}_{3}\end{array}\right]$,

$\boldsymbol{\xi}_{i}=\left[\begin{array}{c}\omega_{i} \\ v_{i}^{x} \\ v_{i}^{y}\end{array}\right]$ and $\quad \boldsymbol{\zeta}_{i}=\left[\begin{array}{c}f_{t i} \\ f_{n i} \\ \tau_{i}\end{array}\right]$

$t$ is the vector composed of the input torques applied on the $i$-th joint $(i=1,2,3)$. It is to be understood that joint 1 refers to the joint of the finger linking the latter to the palm of the hand and that joint 3 links the distal phalanx to the medial phalanx. $\omega_{a}$ is the vector composed of the angular velocities of the $i$-th joint $(i=1,2,3) . \dot{\theta}_{a}$ is the angular velocity of the actuation. $\boldsymbol{\xi}_{i}$ is the twist associated to the $i$-th point of contact and $\zeta_{i}$ is the corresponding wrench. They are operated with the reciprocal product, $\circ$ in the plane containing the finger. For simplicity, we assume that each phalanx is in contact with the object being grasped and that the $i$-th contact can be considered as a point. The twist is composed of the angular velocity, $\omega_{i}$, the velocity components, $v_{i}^{x}$ and $v_{i}^{y}$, of the $i$-th point of contact. These velocity components are in relation of the local reference frame of the $i$-th phalanx. The wrench is composed of the forces acting at the $i$-th contact point: a tangential force, $f_{t i}$, a normal force, $f_{n i}$, and a torque, $\tau_{i}$.

As shown in Hunt (1978), we can define $\boldsymbol{\xi}_{i}$ in the following alternative form:

$\boldsymbol{\xi}_{i}=\sum_{k=1}^{i} \dot{\theta}_{k}\left[\begin{array}{c}1 \\ \mathbf{E} \boldsymbol{r}_{i}^{O_{k}}\end{array}\right]$

Here, $r_{i}^{O_{k}}$ is a vector from $O_{k}$ to the $i$-th point of contact and $\mathbf{E}$ is the transformation matrix for a cross product in the plane:

$\mathbf{E}=\left[\begin{array}{cc}0 & -1 \\ 1 & 0\end{array}\right]$

Now, we can express the right-hand side of Eq. (1) as follows:

$\sum_{i=1}^{3} \xi_{i} \circ \zeta_{i}=\sum_{i=1}^{3} \sum_{k=1}^{i} \dot{\theta}_{k}\left[\begin{array}{c}1 \\ \mathbf{E} \boldsymbol{r}_{i}^{O_{k}}\end{array}\right] \circ \zeta_{i}$

In this article, we make the assumption that the finger is free of all external forces but the torques needed to obtain static equilibrium. Therefore, the wrench is expressed as:

$\zeta_{i}=\left[\begin{array}{c}0 \\ 0 \\ \tau_{i}\end{array}\right]$

And Eq. (4) becomes:

$$
\begin{aligned}
\sum_{i=1}^{3} \xi_{i} \circ \zeta_{i} & =\sum_{i=1}^{3} \sum_{k=1}^{i} \dot{\theta}_{k}\left[\begin{array}{c}
1 \\
\mathbf{E} \boldsymbol{r}_{i}^{O_{k}}
\end{array}\right] \circ\left[\begin{array}{c}
0 \\
0 \\
\tau_{i}
\end{array}\right] \\
& =\tau^{T} \mathbf{J} \dot{\theta}
\end{aligned}
$$

where

$\mathbf{J}=\left[\begin{array}{lll}1 & 0 & 0 \\ 1 & 1 & 0 \\ 1 & 1 & 1\end{array}\right]$

$\mathbf{J}$ is a transformation matrix which links the global torques, $\tau_{i}(\mathrm{i}=1,2,3)$ and the local torques, $\tau_{i}^{*}(\mathrm{i}=1,2,3)$ :

$\tau^{* T}=\tau^{T} \mathbf{J}$

$\tau_{i}^{*}$ is important since it is the amount of torque felt at the $i$-th joint, $i=1,2,3$.

Combining Eqs. (5), (6) with (1), we get:

$$
\begin{aligned}
t^{T} \boldsymbol{\omega}_{a} & =\tau^{T} \mathbf{J} \dot{\theta} \\
& =\tau^{* T} \dot{\boldsymbol{\theta}}
\end{aligned}
$$

At this point, we define $\mathbf{T}$, the transmission matrix that relates vector $\omega_{a}$ to vector $\dot{\boldsymbol{\theta}}$. Explicitly,

$$
\left[\begin{array}{c}
\dot{\theta}_{1} \\
\dot{\theta}_{2} \\
\dot{\theta}_{3}
\end{array}\right]=\left[\begin{array}{ccc}
X_{1} & X_{2} & X_{3} \\
0 & 1 & 0 \\
0 & 0 & 1
\end{array}\right]\left[\begin{array}{c}
\dot{\theta}_{a} \\
\dot{\theta}_{2} \\
\dot{\theta}_{3}
\end{array}\right]
$$




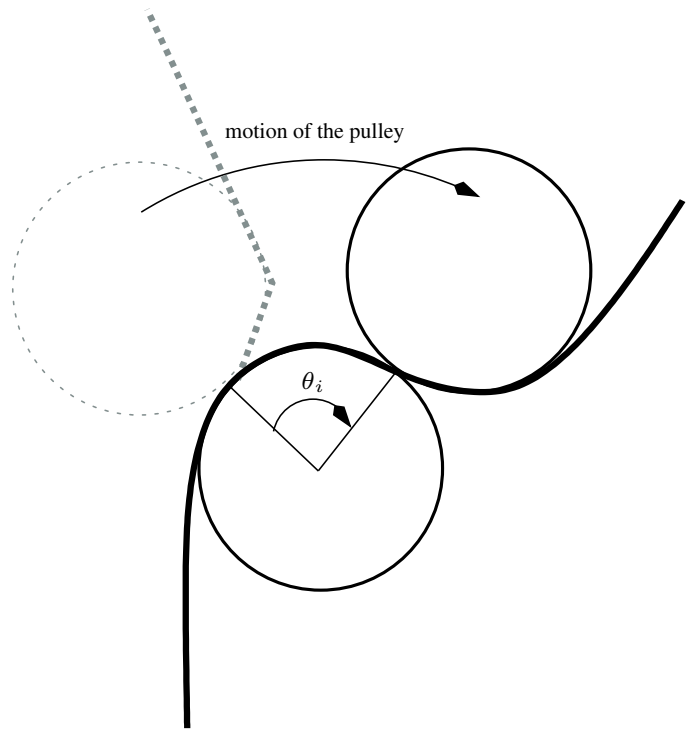

Figure 2. Isolated rotation of a joint.

or

$\dot{\boldsymbol{\theta}}=\mathbf{T} \omega_{a}$

Thus, combining Eqs. (8) and (9), we obtain

$t^{T} \omega_{a}=\tau^{* T} \mathbf{T} \omega_{a}$

which is equivalent to writing

$\tau^{*}=\mathbf{T}^{-T} \mathbf{t}$

\section{Calculation of the transmission matrix for the actuation}

In this section, we take a look at the actuation mechanism of the finger. The mechanism consists of a string attached to the distal phalanx. The string is pulled through a pulley network which produces the joint actuation. The transmission matrix is the mathematical tool that models the behaviour of the joints.

As stated in the last section, the transmission matrix is the application that transforms the actuation velocity vector, $\omega_{a}$, into the time derivatives of the joints coordinates, Eq. (9). The main relation to solve is

$\dot{\theta}_{1}=X_{1} \dot{\theta}_{a}+X_{2} \dot{\theta}_{2}+X_{3} \dot{\theta}_{3}$

First, as illustrated in Fig. 2, we notice that the isolated rotation of each joint, in joint coordinates, results in an elongation of the tendon, $x_{i}$, of the order of the radius of the joint times the rotation of the same joint:

$x_{i}=r_{i a} \Delta \theta_{i} \quad i=1,2,3$
In the case of tendon and pulleys, the total amount of elongation of the tendon corresponds to the rotation of actuation joint times the radius of joint 1 :

$x=r_{1 a} \Delta \theta_{a}$

And because we assume that the tendon is rigid, the total amount of elongation is the sum of the elongation for each joint rotation:

$x=x_{1}+x_{2}+x_{3}$

and by combining Eqs. (13) and (14), we obtain

$r_{1 a} \Delta \theta_{a}=r_{1 a} \Delta \theta_{1}+r_{3 a} \Delta \theta_{2}+r_{5 a} \Delta \theta_{3}$

Now, we differentiate Eq. (15) with respect to time, which gives us

$r_{1 a} \dot{\theta}_{a}=r_{1 a} \dot{\theta}_{1}+r_{3 a} \dot{\theta}_{2}+r_{5 a} \dot{\theta}_{3}$

and reorganize in a form similar to Eq. (12):

$r_{1 a} \dot{\theta}_{1}=r_{1 a} \dot{\theta}_{a}-r_{3 a} \dot{\theta}_{2}-r_{5 a} \dot{\theta}_{3}$

or

$\dot{\theta}_{1}=\dot{\theta}_{a}-\frac{r_{3 a}}{r_{1 a}} \dot{\theta}_{2}-\frac{r_{5 a}}{r_{1 a}} \dot{\theta}_{3}$

So, from Eq. (16), we can define our transmission matrix for the actuation as the following:

$\mathbf{T}_{a}=\left[\begin{array}{ccc}1 & -\frac{r_{3 a}}{r_{1 a}} & -\frac{r_{5 a}}{r_{1 a}} \\ 0 & 1 & 0 \\ 0 & 0 & 1\end{array}\right]$

\section{Calculation of the transmission matrix for the return}

We now turn our attention to the return mechanism. Similarly to the actuation mechanism, the return mechanism consists of a string attached to the distal phalanx and passed through a system of pulleys. However, the string is attached to a linear spring a the base of the finger thus providing the return force.

As in the case of the actuation, we notice in Fig. 3, that the isolated rotation of each joint, in articulation coordinates, results in an elongation of the tendon, $x_{i}$, of the order of the radius of the joint times the rotation of the same joint:

$x_{i}=r_{i b} \Delta \theta_{i} \quad i=1,2,3$

In the case of tendon and pulleys, the total amount of elongation of the tendon corresponds to the rotation of actuation joint times the radius of joint 1 :

$x=r_{1 b} \Delta \theta_{a}$

And because we assume that the tendon is rigid, the total amount of elongation is the sum of the elongation for each joint rotation:

$x=x_{1}+x_{2}+x_{3}$ 


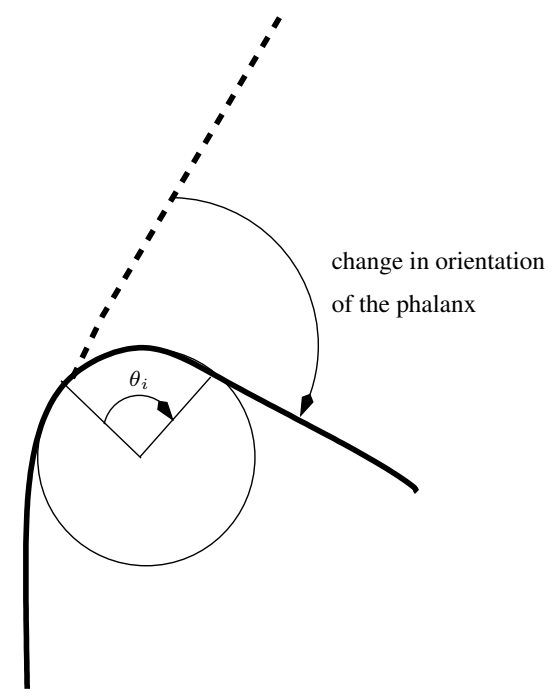

Figure 3. Isolated rotation of a joint for the return mechanism.

and by combining Eqs. (18) and (19), we obtain

$r_{1 b} \Delta \theta_{a}=r_{1 b} \Delta \theta_{1}+r_{3 b} \Delta \theta_{2}+r_{5 b} \Delta \theta_{3}$

Now, we differentiate Eq. (20), which gives us

$r_{1 b} \dot{\theta}_{a}=r_{1 b} \dot{\theta}_{1}+r_{3 b} \dot{\theta}_{2}+r_{5 b} \dot{\theta}_{3}$

and reorganize in a form similar to Eq. (12):

$r_{1 b} \dot{\theta}_{1}=r_{1 b} \dot{\theta}_{a}-r_{3 b} \dot{\theta}_{2}-r_{5 b} \dot{\theta}_{3}$

or

$\dot{\theta}_{1}=\dot{\theta}_{a}-\frac{r_{3 b}}{r_{1 b}} \dot{\theta}_{2}-\frac{r_{5 b}}{r_{1 b}} \dot{\theta}_{3}$

So, from Eq. (21), we can define our transmission matrix for the return as the following:

$\mathbf{T}_{r}=\left[\begin{array}{ccc}1 & -\frac{r_{3 b}}{r_{1 b}} & -\frac{r_{5 b}}{r_{1 b}} \\ 0 & 1 & 0 \\ 0 & 0 & 1\end{array}\right]$

The transmission matrix for the return mechanism is very similar to $\mathbf{T}_{a}$ found for the actuation. However, the pulleys in the return mechanism do not have to be of the same dimensions used for the pulleys in the actuation mechanism. Therefore, we use here the notation " $b$ " to designate a return pulley.

\section{Torques from actuation and return}

In order to find torques due to the underactuated mechanisms, we have found expressions for the transmission matrices. Now we need an expression for the vector $\boldsymbol{t}$ as defined for the use of said vector in Eq. (1). In the actuation process, its expression is straightforward as $\boldsymbol{t}_{\boldsymbol{a}}$ is defined as

$\boldsymbol{t}_{a}=\left[\begin{array}{c}T_{a} \\ 0 \\ 0\end{array}\right]$

where the torque, $T_{a}$ is the cross product of the tension put by the user on the string and the radius of the actuation pulley, $r_{1 a}$. The amplitude is expressed as

$T_{a}=F_{a} r_{1 a}$

Using Eq. (11), we now can get an analytical expression for the torque vector due to the actuation of the finger. This expression is the vector

$\tau_{a}=\left[\begin{array}{c}F_{a} r_{1 a} \\ F_{a} r_{3 a} \\ F_{a} r_{5 a}\end{array}\right]$

We see with Eq. (25) that this design has achieved one of its objectives. The actuation torque at each joint is proportional to the tension given to the string and the actuation torques are not a function of the finger's configuration, i.e., they do not depend on $\theta_{i}, i=1,2,3$.

In the return process, the vector $\boldsymbol{t}_{r}$ is defined in the same fashion as

$\boldsymbol{t}=\left[\begin{array}{c}T_{r} \\ 0 \\ 0\end{array}\right]$

where the torque, $T_{r}$ is the cross product of the tension put by the spring on the string and the radius of the return pulley, $r_{1 b}$. The amplitude is expressed as

$T_{r}=F_{r} r_{1 b}$

Contrary to the case of actuation, the return force, $F_{r}$, is a straightforward and arbitrary value. To express the return force, we must use Hooke's law:

$$
F_{r}=-K y
$$

where $K$ is the spring's stiffness and y, its elongation. Since we use preload in this design, an initial extension $y_{0}$ must be included in the value of $y$. The rest of the value of $y$ is taken into account by the elongation caused by the mouvement of the finger which can be described by using Eq. (20):

$y-y_{0}=r_{1 b} \theta_{1}+r_{3 b} \theta_{2}+r_{5 b} \theta_{3}$

Combining these two expressions, we get a useful expression for the return force:

$F_{r}=-K\left(y_{0}+r_{1 b} \theta_{1}+r_{3 b} \theta_{2}+r_{5 b} \theta_{3}\right)$

We notice that the negative sign in Eq. (28) accounts for the counter-motion characteristic of the return action. 


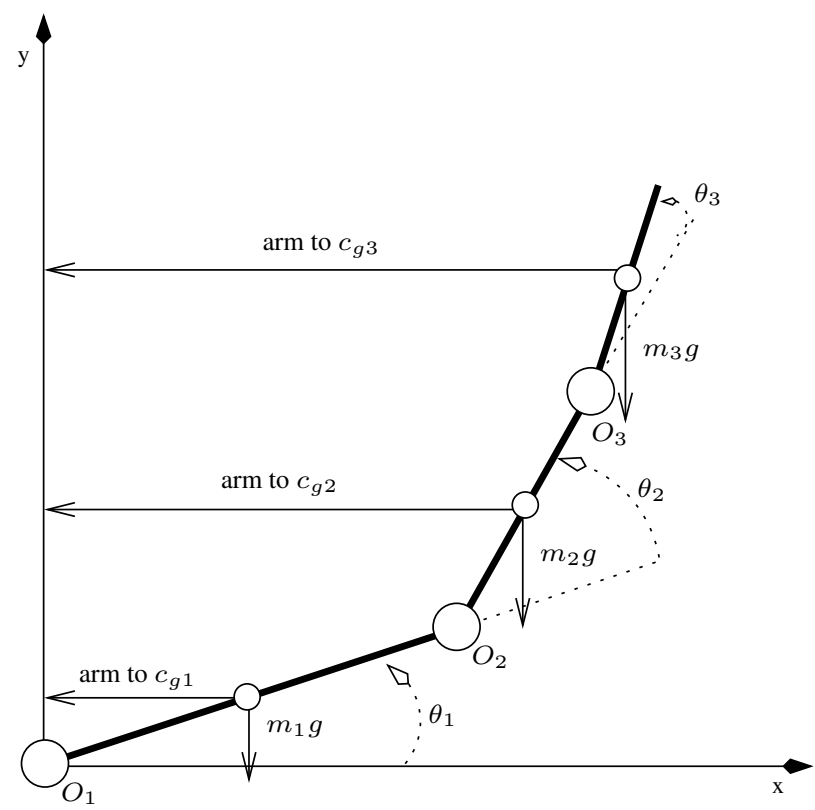

Figure 4. Gravity forces diagram for the proximal joint.

Again, using Eqs. (26), (27) and (28), we now can get an analytical expression for the torque vector due to the return action of the finger. This expression is the vector

$\boldsymbol{\tau}_{r}=\left[\begin{array}{l}-K r_{1 b}\left(y_{0}+r_{1 b} \theta_{1}+r_{3 b} \theta_{2}+r_{5 b} \theta_{3}\right) \\ -K r_{3 b}\left(y_{0}+r_{1 b} \theta_{1}+r_{3 b} \theta_{2}+r_{5 b} \theta_{3}\right) \\ -K r_{5 b}\left(y_{0}+r_{1 b} \theta_{1}+r_{3 b} \theta_{2}+r_{5 b} \theta_{3}\right)\end{array}\right]$

\section{Gravity}

In order to describe the effect of gravity, we must include the spatial orientation of the hand. For this purpose, we will use the Tait-Bryan angles which are a specific convention of Euler angles. These angles correspond to the $X Y Z$ convention where $\varphi$ is the rotation angle around the $\mathrm{X}$-axis, $\theta$ is the rotation angle around the y-axis and, $\psi$ is the rotation angle around the z-axis.

We assume that the hand is palm up and along the Y-axis. The origin of the global coordinate system is at the wrist. This leads to:

- $\theta_{0}$ expresses the angle formed by the palm and the $y-$ axis;

- $\theta_{1}$ expresses the angle formed by the proximal phalanx and the extension of the palm;

- $\theta_{2}$ expresses the angle formed by the medial phalanx and the extension of the proximal phalanx;

- $\theta_{3}$ expresses the angle formed by the distal phalanx and the extension of the medial phalanx;

$-\varphi$ expresses the "roll" of the hand (side-to-side rotation).
Now that the hand is spatially oriented, we use the free body diagram technique to calculate the torques.

We begin to look at the distal phalanx. It is elementary to see that the resulting torque on the distal joint is the cross product of the lever arm from the joint to the centre of mass and the gravity force, the amplitude being

$\tau_{g 3}=\cos (\varphi)\left(m_{3} g c_{g 3} \cos \left(\theta_{0}+\theta_{1}+\theta_{2}+\theta_{3}\right)\right)$

where $g$ is the gravitational acceleration and $m_{i}$ is the mass of the $i$-th phalanx. As for the medial joint, the resulting torque is the sum of the cross products of, on one hand, the arm created by its centre of mass and the gravity force due to the mass of the medial phalanx and, on the other hand, the arm created by its centre of mass and the gravity force due to the mass of the distal phalanx. The amplitude is

$$
\begin{aligned}
\tau_{g 2} & =\cos (\varphi)\left(\left[m_{2} g c_{g 2} \cos \left(\theta_{0}+\theta_{1}+\theta_{2}\right)\right]\right. \\
& +\left[m _ { 3 } g \left(c_{g 3} \cos \left(\theta_{0}+\theta_{1}+\theta_{2}+\theta_{3}\right)\right.\right. \\
& \left.\left.\left.+l_{2} \cos \left(\theta_{0}+\theta_{1}+\theta_{2}\right)\right)\right]\right)
\end{aligned}
$$

Finally, Fig. 4 shows that for the proximal joint, the resulting torque is the sum of the cross products of: the arm created by its centre of mass and the gravity force due to the mass of the proximal phalanx, the arm created by its centre of mass and the gravity force due to the mass of the medial phalanx and, the arm created by its centre of mass and the gravity force due to the mass of the distal phalanx. The amplitude is

$$
\begin{aligned}
\tau_{g 1} & =\cos (\varphi)\left(\left[m_{1} g c_{g 1} \cos \left(\theta_{0}+\theta_{1}\right)\right]\right. \\
& +\left[m_{2} g\left(c_{g 2} \cos \left(\theta_{0}+\theta_{1}+\theta_{2}\right)+l_{1} \cos \left(\theta_{0}+\theta_{1}\right)\right)\right] \\
& +\left[m _ { 3 } g \left(c_{g 3} \cos \left(\theta_{0}+\theta_{1}+\theta_{2}+\theta_{3}\right)+l_{2} \cos \left(\theta_{0}+\theta_{1}+\theta_{2}\right)\right.\right. \\
& \left.\left.\left.+l_{1} \cos \left(\theta_{0}+\theta_{1}\right)\right)\right]\right)
\end{aligned}
$$

In summary, the action of gravity can be modeled by

$\boldsymbol{\tau}_{g}=g \mathbf{C L m} \cos \varphi$

where,

$\mathbf{C}=\left[\begin{array}{ccc}\cos \left(\theta_{0}+\theta_{1}\right) & \cos \left(\theta_{0}+\theta_{1}+\theta_{2}\right) & \cos \left(\theta_{0}+\theta_{1}+\theta_{2}+\theta_{3}\right) \\ 0 & \cos \left(\theta_{0}+\theta_{1}+\theta_{2}\right) & \cos \left(\theta_{0}+\theta_{1}+\theta_{2}+\theta_{3}\right) \\ 0 & 0 & \cos \left(\theta_{0}+\theta_{1}+\theta_{2}+\theta_{3}\right)\end{array}\right]$

is the matrix of the finger's configuration. And,

$\mathbf{L}=\left[\begin{array}{ccc}c_{g 1} & l_{1} & l_{1} \\ 0 & c_{g 2} & l_{2} \\ 0 & 0 & c_{g 3}\end{array}\right]$

is the matrix of geometric parameters. And,

$\boldsymbol{m}=\left[\begin{array}{l}m_{1} \\ m_{2} \\ m_{3}\end{array}\right]$

is the vector of the phalanx masses. The orientation of the palm is simply determined by the sign of the constant $g$. As a convention, $g$ is negative for palm up, positive for palm down. We can also eliminate the effect of gravity by setting the gravitational acceleration to 0 . 
Table 1. Prototype physical parameters.

\begin{tabular}{ccccccc}
\hline & $\mathrm{i}$ & $r_{i a}$ & $r_{i b}$ & $l_{i}$ & $m_{i}$ & $c_{g i}$ \\
\hline Proximal & 1 & 6 & 2.95 & 45 & 0.87 & 21.65 \\
Medial & 2 & 2.75 & 2.75 & 25 & 0.5 & 9.19 \\
Distal & 3 & 1.5 & 2 & n/a & 0.45 & 10.85 \\
\hline
\end{tabular}

\section{The Model}

We now have all the pieces of the puzzle. After the development above, it is natural to conclude that the vector representing the total torque at each joint of the finger is the sum of the expressions given in Eqs. (25), (29) and (33). Allen-Demers et al. (2009) shows a similar result.

The torque vector composed of the torque at each joint is expressed as

$\boldsymbol{\tau}^{*}=\boldsymbol{\tau}_{a}+\boldsymbol{\tau}_{r}+\boldsymbol{\tau}_{g}$

If the reader refers to the beginning of this section, there is one component missing from Eq. (34), namely the torque due to friction. Eq. (34) can be used to simulate the overall behaviour of the finger since friction is negligeable for our purposes. But manufacturing affects the amount of friction in the systems, so the torque due to friction must be obtained empirically. So a corrected model can be written as

$\tau^{*}=\tau_{a}+\tau_{r}+\tau_{g}+\tau_{f}$

\section{Numerical simulations}

Equation (34) allows the simulation of the behaviour of the finger under arbitrary physical parameters. One can study the behaviour of the finger, more precisely the closing sequence, according to the stiffness of the return spring, to the imposed preload, to the orientation of the finger (i.e. palm up or down). Another effect of a physical parameter that needs to be studied is the combination of pulleys of different radii and how it affects the closing sequence and ultimately, the grasping force that can be provided.

The numerical software, Matlab, is used to perform numerical simulations. Equation (34) is programmed and the physical parameters of the prototype of the finger are inserted, as shown in Table 1. The routine sets the configuration of the finger as being flat, i.e., all angles to be zero. An arbitrary and progressively increasing cable tension is applied and once one of the joints' torque becomes positive, the variable $\theta_{i}$ is progressively incremented until the joint angle is $90^{\circ}$ from its initial position.

Figure 5 shows the result of this numerical simulation. We first notice that initially, the torques are negative for all three joints. There is no movement since the joints are designed

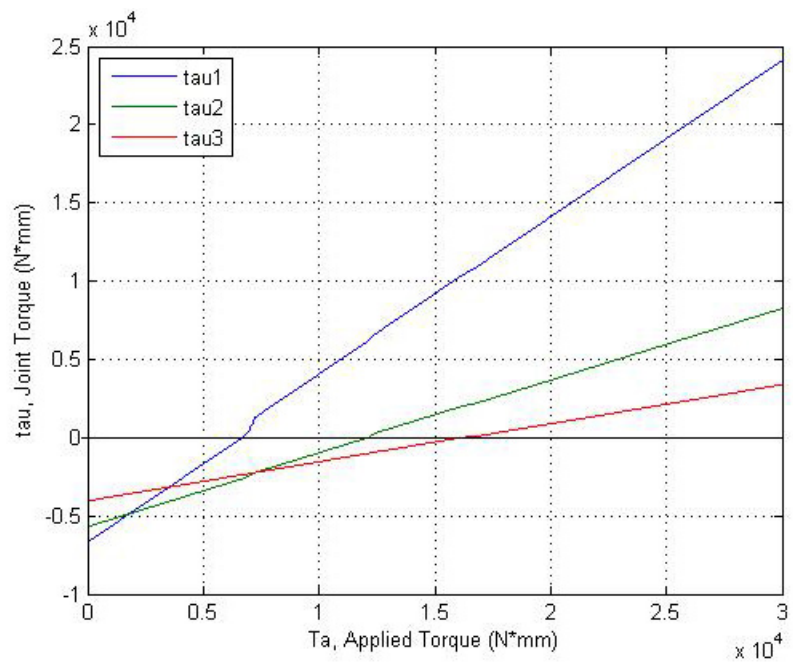

Figure 5. Closing sequence for the finger prototype.

such that they only move in a positive range of angles. Physically, the range in which the torques are negative corresponds to the situation where the actuation does not overcome the return forces, in this case provided by the return spring and gravity.

We also see that the torque for each joint becomes positive at three distinct points. Furthermore, the torques become positive in numerical order such that joint 1 moves before joint 2 which moves before joint 3 . This shows that the design attained another of its objectives which is its closing sequence. We have here a closing sequence where the proximal joint moves fully through its range of motion then the medial joint moves through its range of motion and then the distal joint moves through its range of motion, all seperately. This simulation is consistent with the physical motion of the prototype whose paramters were used for the simulation.

Figure 6 shows the simulation with a small preload, smaller than 10 . We see the same general features that were observed in Fig. 5. However, the negative tension zone is much smaller. This is no surprise since a smaller preload leads to a smaller return force and so a smaller actuation force is needed to overcome the former forces.

Also, we observe that the joint torques still become positive in numerical order. However, the crossing points are very close to each other which suggests a closing sequence that will not follow that crossing order. Multiple joints will move at the same time and so the closing sequence will be uncontrolled.

Finally, Fig. 7 shows a simulation where there is no return. This simulation shows that the closing sequence does not correspond to the design goal. Since there are no return forces and so only gravity forces oppose the actuation, we can see that the distal joint will close first since it is lighter. The proximal joint will be last since it is opposed to the finger's total mass. 


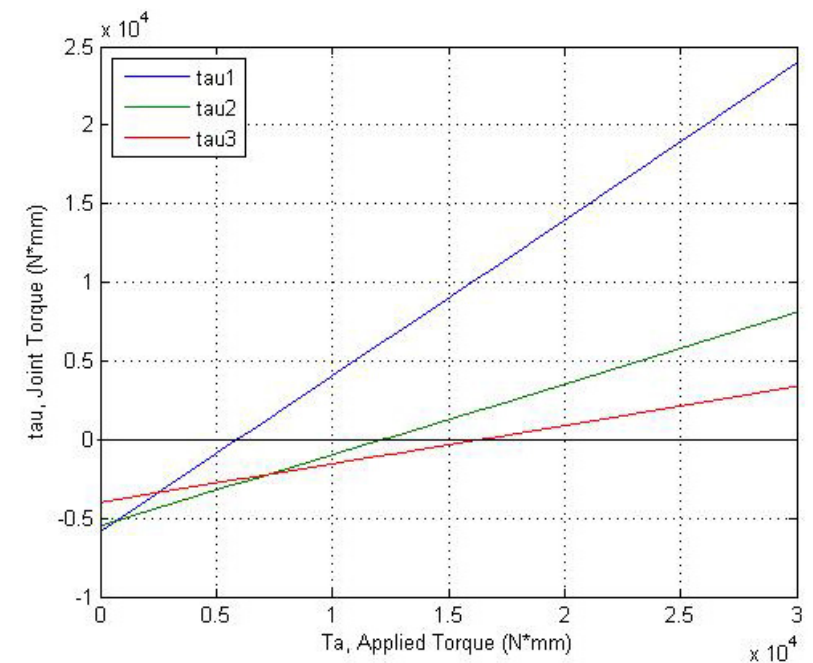

Figure 6. Closing sequence with small preload.

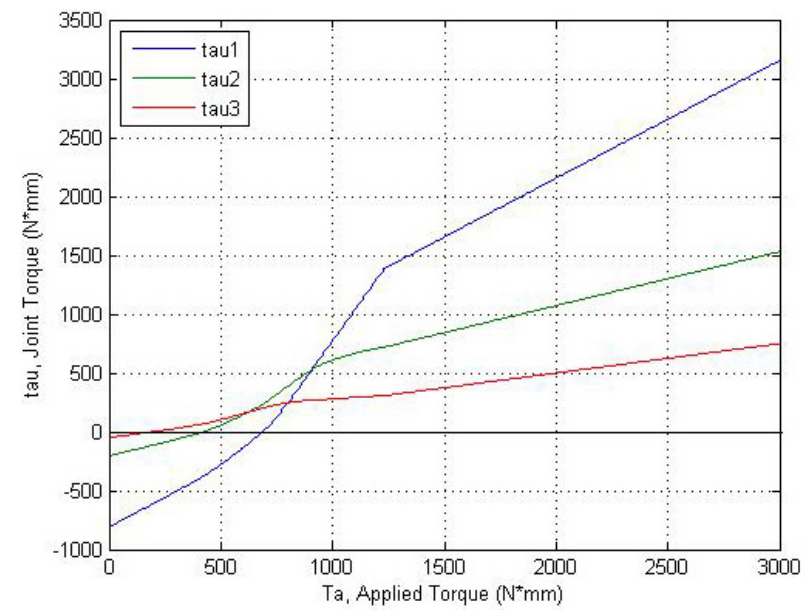

Figure 7. Closing sequence with no return.

10

\section{Conclusions}

The model presented in this paper is simple and flexible. It expands the work in Birglen et al. (2008); Allen-Demers et al. (2009) by adapting the equations to the specific case of human-like fingers. It also completes said work by adding the effect of physical forces such as gravity and that, in any spatial orientation of the hand. Furthermore, each force can be easily isolated. Each joint can be also isolated. Hence, the influence of the type of force on a specific joint can be simulated to study its effect along the full range of motion. Each physical parameter is also easily isolated. The effect of combinations of pulley ratios can be easily studied.

This leads to a better understanding and characterisation of the intelligence of the mechanism or of the effect of the return force. Such characterisations can help with the optimisation of the design.
Even though this model was focusing on the internal torques acting on the individual joint, it can be easily extended to external forces. A stability characterisation is needed to see the adaptability of the design.

Acknowledgements. The authors would like to acknowledge the financial support of the Natural Sciences and Engineering Research Council of Canada (NSERC) and of the Canada Research Chair program.

Edited by: J. L. Herder

Reviewed by: two anonymous referees

\section{References}

Allen-Demers, L. and Gosselin, C.: Kinematic Design of an Ejection-Free Underactuated Anthropomorphic Finger, Proceedings of the 2009 IEEE International Conference on Robotics and Automation, 2009.

Belfiore, M.: The Department of Mad Scientists; How DARPA Is Remaking Our World, from the Internet to Artificial Limbs, Smithsonian Books, New York, NY, 2009.

Birglen, L., Laliberte, T., and Gosselin, C.: Underactuated Robotic Hands, Springer, Berlin, Germany, 2008.

Hunt, K.: Kinematic Geometry of Mechanisms, Oxford University Press, Oxford, England, 1978. 